\title{
$B R C A 1 / 2$ mutation testing in breast cancer patients: a prospective study of the long-term psychological impact of approach during adjuvant radiotherapy
}

\author{
Kathryn J. Schlich-Bakker - Margreet G. E. M. Ausems - Maria Schipper • \\ Herman F. J. ten Kroode · Carla C. Wárlám-Rodenhuis · Jan van den Bout
}

Received: 27 June 2007 / Accepted: 9 July 2007/Published online: 3 August 2007

(C) Springer Science+Business Media, LLC 2007

\begin{abstract}
This study assessed psychological distress during the first year after diagnosis in breast cancer patients approached for genetic counseling at the start of adjuvant radiotherapy and identified those vulnerable to long-term high distress. Of the approached patients some chose to receive a DNA test result $(n=58)$, some were approached but did not fulfill criteria for referral $(n=118)$ and some declined counseling and/or testing $(n=44)$. The comparative group consisted of patients not eligible for genetic counseling $(n=182)$ and was therefore not approached. Patients actively approached for genetic counseling showed no more long-term distress than patients not eligible for such counseling. There were no differences between the subgroups of approached patients. Predictors for long-term high distress or an increase in distress over time were pre-existing high distress and a low quality of life, having children, and having no family members with
\end{abstract}

K. J. Schlich-Bakker $(\bowtie)$ · H. F. J. ten Kroode

Department of Medical Oncology, University Medical Centre Utrecht, P.O. Box 85060, Utrecht 3508 AB, The Netherlands e-mail: K.J.Schlich@umcutrecht.nl

M. G. E. M. Ausems

Department of Medical Genetics, University Medical Centre Utrecht, Utrecht, The Netherlands

\section{Schipper}

Centre of Biostatistics, Utrecht University, Utrecht,

The Netherlands

\section{C. Wárlám-Rodenhuis}

Department of Radiation Oncology, University Medical Centre Utrecht, Utrecht, The Netherlands

J. van den Bout

Department of Clinical and Health Psychology, Utrecht

University, Utrecht, The Netherlands breast cancer. It is concluded that breast cancer patients can be systematically screened and approached for genetic counseling during adjuvant radiotherapy without imposing extra psychological burden. Patients vulnerable to longterm high distress already displayed high distress shortly after diagnosis with no influence of their medical treatment on their level of distress at long-term.

Keywords BRCA1/2 mutation searching - Genetic counseling · Long-term psychological impact · Breast cancer patients

\section{Introduction}

Breast cancer is among the most widely diagnosed cancers in the world with an estimated 1.15 million cases worldwide in 2002 [1]. The psychological effect of a breast cancer diagnosis and subsequent treatment is well documented, revealing high distress in 20-30\% of breast cancer patients during the first year after diagnosis. The majority no longer experience serious psychological distress one year after surgery [2-6]. Patient-related factors such as age, personality characteristics, previous psychological treatment, severely stressful non-cancer life experiences, preexisting general health complaints, illness perceptions and postoperative distress are all related to long-term psychological distress. Objective cancer-related factors such as TNM-stage and type of local or adjuvant treatment do not influence psychological distress after diagnosis [3-7].

An estimated $5-10 \%$ of all breast cancers may be accounted for by inherited autosomal dominant susceptibility genes [8] accompanied by an increased risk for a second breast and/or ovarian cancer [9]. Pre-symptomatic DNA testing in families with a $B R C A$ mutation is associated 
with psychological benefits for non-carriers while, generally, no adverse psychological effects are observed amongst carriers [10]. However, some characteristics of applicants vulnerable to high distress have been identified including: high pre-test distress, carrier status, complicated grief, more affected first-degree relatives, and strong emotional illness representations [11, 12].

Among breast cancer patients diagnosed between 4 months and 32 years before undergoing genetic testing, those diagnosed within the year prior to testing were more anxious, reporting more breast-cancer-specific distress than those with more than one year between diagnosis and testing [13]. Affected mutation carriers, especially recently diagnosed patients, showed a stronger decline in psychological well-being than unaffected mutation carriers two weeks after the DNA result [14]. This potential negative impact was also reported by Bonadona et al. [15].

A systematic active approach of recently diagnosed breast cancer patients for BRCAl/2 mutation screening increases patients' awareness of genetic issues and may positively influence the actual uptake for BRCAl/2 mutation searching. Consequently, breast cancer patients are included who might not have applied for genetic counseling on their own initiative and who might show less psychological resilience than the previously studied non-affected participants who applied on their own initiative [16]. The future role of genetic counseling and BRCA testing during the process of deciding on primary surgical treatment for breast cancer [17-20] implies the time between diagnosis and genetic counseling and testing will only become shorter. Breast cancer patients will be approached for genetic counseling during the most stressful period of treatment, i.e. immediately after diagnosis.

Earlier we reported on the short-term psychological responses of breast cancer patients to an active approach for genetic counseling seven weeks after surgical treatment, at the beginning of their adjuvant radiotherapy [21]. We found no increase in psychological distress shortly after such an approach. Nor was there a difference in the level or course of psychological distress between patients approached for genetic counseling and those not eligible and therefore not approached. However, of all approached breast cancer patients, $50 \%$ showed high distress after approach. Approached patients vulnerable to short-term high distress were younger, single, less optimistic, experienced little social support, used an avoiding coping style and displayed a lower quality of life and higher distress level before approach for genetic counseling. To the best of our knowledge no studies have reported on the long-term effects of such a pro-active approach in such an early stage of treatment.

The aim of this study was to gain insight into the course of psychological distress during the year following breast cancer diagnosis in: (1) patients who receive a DNA-test result, (2) patients who initially take part in genetic counseling but who do not fulfill criteria for further counseling, and (3) patients who decline genetic counseling and/or testing. A second aim was to identify breast cancer patients vulnerable to long-term high distress after being approached for genetic counseling shortly after diagnosis.

According to a retrospective study among breast cancer patients who received their carrier status some 2 months to 10 years after their cancer diagnosis, the majority believed there was already an emotional overload in coping with the diagnosis and treatment, and that offering DNA testing shortly after learning they had cancer would have added too much additional stress [22]. The majority of health professionals were of the same opinion [22]. This report gave us reason to expect a higher long-term psychological distress among recently diagnosed breast cancer patients approached for genetic counseling and BRCA testing compared to patients not approached for counseling. We expected the same factors to influence long-term levels of distress as identified for short-term distress (see above) [21]. Additionally, we expected highly distressed patients to have more relatives with breast cancer $[12,23]$.

\section{Patients and methods}

\section{Participants}

Participants were breast cancer patients referred to the University Medical Centre Utrecht for adjuvant radiotherapy between January 2002 and March 2004. Inclusion criteria were: a first diagnosis of breast cancer, age between 18 and 75 years, and fluent in the Dutch language.

\section{Procedure}

The procedure used in our initial approach for genetic counseling has been described elsewhere [21]. Referred breast cancer patients were recruited for our psychological study prior to their first visit to the Department of Radiotherapy. During this first visit they were assessed for eligibility for genetic counseling using factors presumed predictive for hereditary breast cancer [21]. If patients were positive for at least one factor, they were actively approached to have a family pedigree drawn up ( = initial approach). If this revealed at least one criterion for further genetic counseling patients were referred to the Department of Medical Genetics for further counseling and $B R C A$ $1 / 2$ mutation testing. The criteria were: (1) breast cancer in patient or relative $<40$ years of age, (2) two or more relatives with breast cancer, (3) multifocal, multicentric or bilateral breast cancer in patient or relative with the first 
breast cancer diagnosed $<50$ years of age, (4) ovarian cancer in patient or relative, and (5) a male relative with breast cancer.

Patients completed questionnaires one week prior to approach for genetic counseling (T0), four weeks after initial approach (T1), three weeks after pedigree compilation (T2), three weeks after the first visit to the Department of Medical Genetics (T3), and three weeks after receiving DNA test results (T4) (Fig. 1). Questionnaires were returned by mail. All participants completed T0 and T1. Where the comparative group completed all five questionnaires, those approached for counseling only completed three questionnaires in total. The timing of the third questionnaire depended on when a patient left the genetic counseling protocol, either due to ineligibility for further counseling, after declining further counseling or after receiving a DNA test result. This study was approved by the Medical Ethics Committee of the University Medical Centre Utrecht.

\section{Measures}

At baseline (T0) participants provided demographics (age, marital status, number of children, educational level and employment), information on family cases of breast cancer and on their involvement with cancer in relatives. The treating physician registered medical history including time since surgery, type of surgery (mastectomy vs. lumpectomy), extent of adjuvant therapy and TNM stage.

Participants completed the following psychological characteristics:

Fig. 1 Study flow scheme

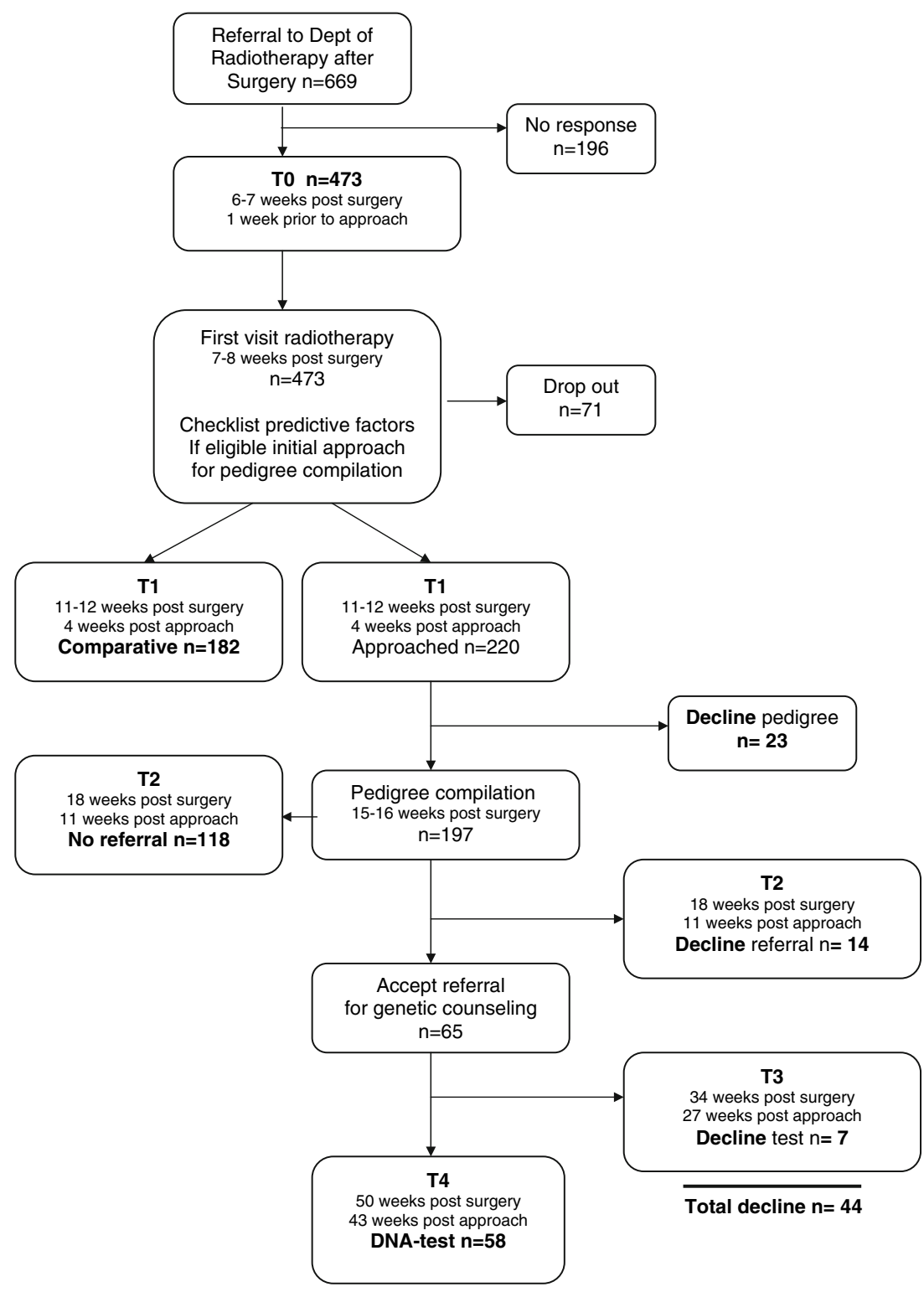


- Utrecht Coping List (UCL) [24], a 15-item coping strategy scale evaluating active coping, social support seeking, avoidance and palliative reactions on a fourpoint frequency scale from 'almost never' to 'nearly always' (Cronbach's alpha was $0.67-0.84$ in a random selection of Dutch cancer patients).

- The Optimism part of the Self-Assessment Questionnaire-Nijmegen $(S A Q-N)[25,26]$, an 8-item scale measuring the level of optimistic outlook on life on a four-point frequency scale ranging from 'almost never' to 'nearly always'.

- The Quality of Life part of the EORTC-QLQ [27], a 2item subscale measuring general quality of life in cancer patients on a seven-point Likert scale.

- A self-designed single item on social support, scoring whether patients have someone to share personal problems and feelings with ('no', 'yes, with one person', and 'yes, with more than one person').

At each measurement (T0 through T4) participants completed the following two outcome measures:

- Hospital Anxiety and Depression Scale (HADS) [28], a 14-item scale measuring anxiety and depression (7 items each) with a total score ranging from 0 to 42 . A total score $>13$ is considered indicative of adjustment disorder [29].

- Impact of Event Scale (IES) [30], a 15-item scale measuring intrusion (7 items) and avoidance (8 items) geared towards breast cancer as the distressing event. A total score $\geq 26$ is considered indicative of clinical adaptation difficulties [31, 32].

To identify vulnerable patients, the total HADS and IES scores at each measurement were dichotomized using the cut-off scores for high psychological distress (HADS $>13$, IES $\geq 26$ ). The development of both measures was identified for each patient, yielding four patterns: (1) patients scoring under the cut-off at baseline and at the last measurement (low group), (2) patients scoring under the cut-off at baseline and above the cut-off at the last measurement (increasing group), (3) patients scoring above the cut-off at baseline but drop below at the last measurement (decrease group), and (4) patients scoring above the cut-off at baseline and at the last measurement (high group). The high and increase groups were considered vulnerable.

\section{Statistical analysis}

Using mixed models in the statistical program $\mathrm{R}$ (version 2.1.0.) accounting for the multiple measurement of each individual patient and correcting for differences in medical history, we analyzed courses of psychological distress and whether distress depends on approach for genetic counseling and/or participation in DNA testing. Based on dichotomized baseline scores for psychological distress, the two low baseline groups and the two high baseline groups were compared on demographic characteristics, medical history and psychological measures with non-parametric tests using the Statistical Package for Social Sciences (SPSS 12.0). Differences in univariate variables were analyzed using the $\chi^{2}$-test on nominal variables and the Mann-Whitney test on continuous variables. To identify predictors for long-term high psychological distress, these variables were entered into a binary logistic regression model using a forward, stepwise, procedure model building strategy (Nagelkerke's $R^{2}$ ).

\section{Results}

Characteristics of the study groups

Of the 669 breast cancer patients recruited for this psychological study $473(71 \%)$ returned the baseline questionnaire. Of the $473,402(85 \%)$ patients completed at least two measurements and were included in the study; $348(74 \%)$ patients completed all measurements. Figure 1 shows participant details divided into four groups. Fiftyeight patients received a DNA test result (DNA test group), while the pedigree analyses showed 118 patients did not meet criteria for further counseling (No referral group). Patients who declined genetic counseling at any time were grouped into the Decline group $(n=44)$. One hundred and eighty-two patients were not eligible for genetic counseling and were therefore not approached (Comparative group). Differences between the total group approached and the comparative group have been described elsewhere [21]. Table 1 gives the demographic characteristics and medical history of the four groups.

Level and course of psychological distress

The four groups did not differ in level or course of general anxiety and depression or breast-cancer-specific distress. Nor did they differ in the percentage of patients scoring above the cut-off scores for high anxiety and depression or severe adaptation difficulties. Originally the model was corrected for differences in medical history between the four groups. However, no effect was identified proving a simplified model as sufficient for the investigation of the level and course of distress over time. The course of psychological distress in all four groups fits a curvi-linear model, decreasing after the initial approach up to week 27 , followed by a small increase up to week 43 (Figs. 2 and 3). The models that best fit the course of distress were: 
Table 1 Demographic and medical characteristics of breast cancer patients who received a DNA-test result, patients who were not eligible for further counseling, patients who declined genetic counseling, and the comparative group

DNA-test (1) $\quad$ No referral (2) $\quad$ Decline (3) $\quad$ Comparative $\quad \chi^{2} \quad p \quad$ Sign.

$\begin{array}{llllll}(n=58) & (n=118) & (n=44) & (4)(n=182) & & p\end{array} \quad$\begin{tabular}{l} 
Sifferences \\
\hline
\end{tabular}

\begin{tabular}{|c|c|c|c|c|c|c|c|}
\hline \multicolumn{8}{|l|}{ Demographics } \\
\hline Age, mean (range) & $45(24-70)$ & $55(41-74)$ & $49(28-72)$ & $57(40-74)$ & 54.672 & $0.000^{*}$ & $\begin{array}{l}1-2,1-4, \\
3-2,3-4\end{array}$ \\
\hline With partner $(\%)$ & 80.7 & 76.1 & 81.8 & 74.2 & 1.655 & 0.648 & \\
\hline With children $(\%)$ & 86.2 & 82.9 & 70.5 & 82.3 & 4.359 & 0.222 & \\
\hline Education $\geq$ high school $(\%)$ & 49.1 & 41.0 & 59.1 & 29.7 & 16.706 & $0.001^{*}$ & $3-4$ \\
\hline Employed at diagnosis (\%) & 73.7 & 58.1 & 59.1 & 44.5 & 16.920 & $0.001^{*}$ & $1-4$ \\
\hline \multicolumn{8}{|l|}{ Medical history } \\
\hline Days post operation, mean (range) & $58.4(21-201)$ & $55(19-153)$ & $48(13-141)$ & $56(9-182)$ & 2.068 & 0.558 & \\
\hline Mastectomy (\%) & 27.6 & 15.4 & 20.5 & 19.6 & 9.668 & $0.020^{*}$ & $1-4$ \\
\hline \multicolumn{8}{|l|}{ Adjuvant treatment } \\
\hline None $(\%)$ & 46.6 & 57.3 & 45.5 & 70.3 & 16.779 & $0.001^{*}$ & $4-1,4-3$ \\
\hline Chemo prior to RT $(\%)$ & 13.8 & 6.8 & 18.2 & 4.4 & 11.487 & $0.007^{*}$ & $4-3$ \\
\hline Chemo after RT (\%) & 12.1 & 10.3 & 9.1 & 3.8 & 7.374 & 0.054 & \\
\hline Hormone $(\%)$ & 27.6 & 25.6 & 27.3 & 21.4 & 1.639 & 0.656 & \\
\hline \multicolumn{8}{|l|}{ pN stage } \\
\hline 0 & 43.1 & 56.9 & 53.5 & 65.3 & 9.564 & $0.022^{*}$ & $1-4$ \\
\hline 1 & 50.0 & 40.5 & 44.2 & 33.5 & 5.779 & 0.123 & \\
\hline 2 & 5.2 & 0.9 & 2.3 & - & 8.254 & $0.009^{*}$ & $1-4$ \\
\hline 3 & 1.7 & 1.7 & - & 1.1 & 0.942 & 0.921 & \\
\hline \multicolumn{8}{|l|}{ Family history bc } \\
\hline None $(\%)$ & 38.6 & 21.6 & 30.2 & 92.3 & 193.161 & $0.000^{*}$ & $4-1,4-2,4-3$ \\
\hline Only FDR bc (\%) & 21.1 & 22.4 & 27.9 & 1.1 & 52.643 & $0.000^{*}$ & $4-1,4-2,4-3$ \\
\hline Only SDR bc (\%) & 24.6 & 42.2 & 37.2 & 6.0 & 63.727 & $0.000^{*}$ & $4-1,4-2,4-3$ \\
\hline $\mathrm{FDR}+\mathrm{SDR}(\%)$ & 15.8 & 13.8 & 4.7 & 0.5 & 29.947 & $0.000^{*}$ & $4-1,4-2$ \\
\hline Involvement cancer in family (\%) & 33.9 & 37.6 & 25.0 & 28.5 & 3.727 & 0.291 & \\
\hline
\end{tabular}

* Significant difference $p<0.05 ; \mathrm{RT}=$ radiotherapy, $\mathrm{FDR}=$ first degree relative, $\mathrm{SDR}=$ second degree relative, bc $=$ breast cancer, employed $=$ employed outside the home upon diagnosis, $\mathrm{pN}$ stage $=$ pathologic regional lymph node stage according to the UICC TNM classification of malignant tumors, fifth edition

$$
\begin{aligned}
& 9.3314-0.1251 * \text { time }+0.0027 * \text { time }^{2} \text { for anxiety } \\
& \text { and depression (C.I. time }-0.1745 \text { to }-0.0757, \\
& \left.p=0.000 \text { and C.I. time }{ }^{2} 0.0015-0.0038, p=0.000\right) \\
& \text { and } \\
& 25.6297-0.3457 * \text { time }+0.0067 * \text { time }^{2} \text { for breast- } \\
& \text { cancer-specific distress (C.I. time }-0.4443 \text { to }-0.2471 \text {, } \\
& \left.p=0.000 \text { and C.I. time }{ }^{2} 0.0044-0.0090, p=0.000\right) .
\end{aligned}
$$

Predictors for pattern of psychological distress

\section{Anxiety and depression (HADS)}

Total baseline scores of anxiety and depression show a Pearson correlation of 0.72 with total scores on the last measurement. A pattern of anxiety and depression was obtained for 215 of the 220 approached patients. The four patterns did not differ on demographic characteristics or medical history. Compared to the HADS low group, the HADS increase group had a more avoiding coping style $(Z=-2.718, p=0.006)$ and reported lower quality of life $(Z=-3.056, p=0.002)$, more anxiety and depression $(Z=-4.231, p=0.000)$ and more breast-cancer-specific distress $(Z=-2.777, p=0.005)$ at baseline. The baseline level of anxiety and depression was the only predictor for an increase in anxiety and depression at the last measurement (O.R. 1.416, C.I. 1.180-1.700, Table 2).

At baseline the HADS high group was less optimistic $(Z=-2.150, p=0.031)$, experienced a lower quality of life $(Z=-3.272, p=0.001)$ and reported more anxiety and depression $(Z=-2.710, p=0.006)$ than the HADS decrease group. A high pattern of anxiety and depression was best predicted by lower perceived quality of life at 


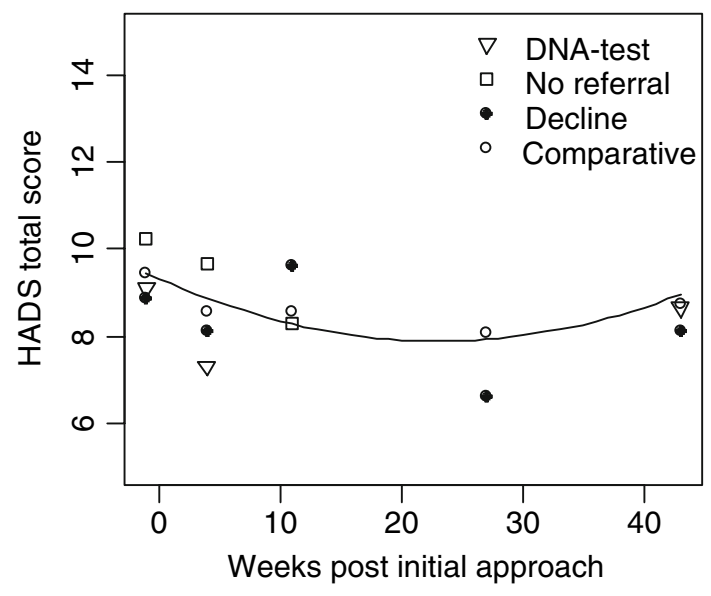

Fig. 2 Fitted model for the course of the total HADS score measured one week prior to approach and 4, 11, 27 and approximately 43 weeks after approach for genetic counseling

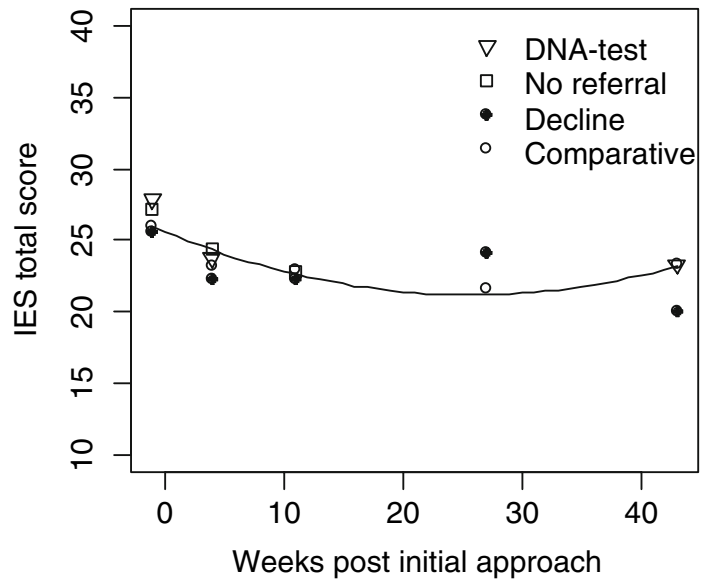

Fig. 3 Fitted model for the course of the total IES score measured 1 week prior to approach and 4,11, 27 and approximately 43 weeks after approach for genetic counseling

baseline (O.R. 0.912, C.I. 1.180-1.700) and having children (O.R. 0.125, C.I. 0.018-0.863, Table 2).

\section{Breast-cancer-specific distress (IES)}

Total baseline scores and last scores on breast-cancer-specific distress showed a Pearson correlation of 0.56. A pattern for breast-cancer-specific distress was obtained for 214 approached patients. There were no differences between the four patterns on demographics or medical history. Compared to the IES low group, the IES increase group demonstrated a more avoiding coping style $(Z=-2.011$, $p=0.044)$ and a higher anxiety and depression $(Z=-2.063$, $p=0.038)$ and breast-cancer-specific distress $(Z=-3.130$, $p=0.001)$ at baseline. An increase in breast-cancer-specific distress was predicted by the level of breast-cancer-specific distress at baseline (O.R. 1.181, C.I. 1.034-1.348, Table 2).
The IES high group reported more anxiety and depression $(Z=-3.400, p=0.001)$ and breast-cancer-specific distress $(Z=-2.562, p=0.010)$ at baseline than the IES decrease group. In addition, the IES high group more often had no breast cancer in the family $\left(\chi^{2}=7.607, p=0.009\right)$ and less often only second-degree relatives with breast cancer $\left(\chi^{2}=9.836, p=0.003\right)$. A high pattern of breast-cancerspecific distress was predicted firstly by high anxiety and depression at baseline (O.R. 1.222, C.I. 1.096-1.363), followed by having no family members with breast cancer, rather than only second-degree affected relatives (O.R. 2.680, C.I. $0.462-15.548)$ or both first- and second-degree relatives (O.R. 0.773, C.I. 0.167-3.588) (Table 2).

\section{Discussion}

Contrary to our expectations there was no adverse effect from actively approaching recently diagnosed breast cancer patients for genetic counseling or from their participation in counseling and DNA testing during primary treatment. Long-term psychological distress scales showed no differences in either of our groups' level or course of psychological distress during the year following diagnosis. The hesitance of health professionals to approach breast cancer patients for genetic testing during primary treatment [22] thus seems unwarranted, even though recently diagnosed patients experience more distress during genetic counseling and testing than patients diagnosed earlier [13]. Perhaps the possibly hereditary nature of their cancer is not as distressing as the diagnosis of breast cancer itself [33].

The possibility of a ceiling effect in distress after diagnosis is not a valid explanation for the absence of adverse effect since there was an increase in distress among a subgroup of patients. This subgroup consisted mainly of patients who reported higher levels of distress prior to approach and included patients not referred for further counseling, patients who declined counseling at some point and some who received a DNA test result.

The curvi-linear course of psychological distress in all groups may relate to primary treatment for breast cancer combined with decreasing distress simply due to passing of time [31]. Highest distress was noted close to diagnosis and surgery, decreasing during adjuvant treatment and reaching the lowest point at the end of radiation therapy. Anticipation of the annual mammogram and fear of a possible recurrence or second breast cancer may account for the small increase towards the end of the first year after diagnosis [34].

Considering earlier findings among breast cancer patients, the present study supports psychological distress at baseline as main predictor for later distress $[5,6]$ and the absence of influence of breast cancer medical treatment 
Table 2 Significant predictors for long-term high psychological distress: forward stepwise binary logistic regression model

$\mathrm{QL}=$ Quality of life,

FDR $=$ first degree relative,

$\mathrm{SDR}=$ second degree relative, $\mathrm{bc}=$ breast cancer

\begin{tabular}{|c|c|c|c|c|}
\hline & $\chi^{2}$ & $P$ & $R^{2}$ & Increase $R^{2}$ \\
\hline \multicolumn{5}{|l|}{$N=215$} \\
\hline \multicolumn{5}{|l|}{ HADS low $n=134(62.3 \%)$} \\
\hline \multicolumn{5}{|l|}{ HADS increase $n=24(11.2 \%)$} \\
\hline Step 1 High HADS baseline & 18.292 & 0.000 & 0.215 & \\
\hline \multicolumn{5}{|l|}{ HADS decrease $n=24(11.2 \%)$} \\
\hline \multicolumn{5}{|l|}{ HADS high $n=33(15.3 \%)$} \\
\hline Step 1 Low QL baseline & 11.891 & 0.001 & 0.291 & \\
\hline Step 2 Having children & 5.065 & 0.024 & 0.395 & 0.104 \\
\hline \multicolumn{5}{|l|}{$N=214$} \\
\hline \multicolumn{5}{|l|}{ IES low $n=87(40.7 \%)$} \\
\hline \multicolumn{5}{|l|}{ IES increase $n=12(5.6 \%)$} \\
\hline Step 1 High IES baseline & 8.422 & 0.004 & 0.183 & \\
\hline \multicolumn{5}{|l|}{ IES decrease $n=35(16.4 \%)$} \\
\hline \multicolumn{5}{|l|}{ IES high $n=80(37.4 \%)$} \\
\hline Step 1 High HADS baseline & 18.745 & 0.000 & 0.242 & \\
\hline Step 2 No family history bc & 10.940 & 0.012 & 0.364 & 0.122 \\
\hline Only FDR & & 0.339 & & \\
\hline Only SDR & & 0.006 & & \\
\hline $\mathrm{FDR}+\mathrm{SDR}$ & & 0.057 & & \\
\hline
\end{tabular}

[3, 4, 6, 7], including approach for genetic counseling. However, our study does not confirm age, marital status and level of optimism as relevant for long-term distress $[4,7,21]$.

Of the short-term psychological distress predictors [21], only the reported quality of life prior to approach continued to play a role in the prediction of long-term distress. We identified new factors such as having children or having no relatives with breast cancer. The role of having children in long-term psychological distress is consistent with other findings [20]. It is possible that during primary treatment patients are overwhelmed by distress of their diagnosis and that only after completing primary treatment do they begin to worry about the implications for their children thus making this a more important and longer-term cause of distress.

The finding that patients with high baseline distress and no relatives with breast cancer are more likely to remain highly distressed does not confirm findings by Van Oostrom et al. [12] among healthy applicants from families with known BRCA1/2 mutations. Besides negative experiences of breast cancer in the family (e.g. extensive treatment or loss of a family member), positive experiences during course of treatment may occur yielding positive effects on psychological distress. The subgroup of patients with high baseline distress and no relatives with breast cancer lack these experiences and therefore this potentially positive effect.

In summary, our study suggests that breast cancer patients can be approached for genetic counseling at the beginning of adjuvant radiotherapy and undergo genetic testing without adding an extra psychological burden. It should be noted that our findings pertain solely to the affected proband. We did not study effects of diagnosis and consecutive genetic testing on family members. Nor did we describe in the current write up the effects on the partners of newly diagnosed patients. Additionally, although patients were approached shortly after diagnosis, genetic counseling and testing took approximately six months to complete. Receiving a DNA result within three to six weeks, as in true rapid testing, may affect the level and course of patients' distress differently. Another limitation was the absence of a true experimental design since the comparative group comprises patients ineligible for genetic counseling and thus not approached.

Based on the present results, clinicians should preferably have knowledge of patients' psychological distress and their perceived quality of life before approaching them for genetic counseling in order to provide adequate support if needed. Assessment should preferably take place using standardized questionnaires, so results can be easily compared to norms. Family history of breast cancer and whether a patient has children should also be noted.

Early detection of BRCA1/2 mutations is playing an increasing role in decisions about surgical treatment $[16,19]$. Patients suspected of hereditary breast cancer who have not reached a decision about definite local treatment may benefit from immediate genetic referral [17]. We have shown that systematic screening with an active approach 
and participation in genetic testing during adjuvant radiotherapy does not influence patients' levels of distress longterm. The next question is what the psychological impact is of having genetic counseling and rapid BRCA results before making surgical decisions.

Acknowledgements Special thanks are in order to all the participating patients and radiation oncologists from the Department of Radiotherapy at the University Medical Centre Utrecht, the Netherlands. We also thank Jeanne van Echtelt for asking all the patients to participate in the study and further monitoring their participation and Jackie Senior and David Alexander for critically reading the manuscript. The Dutch Cancer Society supported this work, grant no. UU 2001-2386.

\section{References}

1. Parkin DM, Bray F, Ferlay J et al (2005) Global cancer statistics, 2002. CA Cancer J Clin 55:74-108

2. Irvine D, Brown B, Crooks D (1991) Psychosocial adjustment in women with breast cancer. Cancer 67:1097-1117

3. Bleiker EM, Pouwer F, van der Ploeg HM et al (2000) Psychological distress two years after diagnosis of breast cancer: frequency and prediction. Patient Educ Couns 40:209-217

4. Burgess C, Cornelius V, Love S et al (2005) Depression and anxiety in women with early breast cancer: five year observational cohort study. Br Med J 330:702-705

5. Millar K, Purushotham AD, McLatchie E et al (2005) A 1-year prospective study of individual variation in distress, and illness perceptions, after treatment for breast cancer. J Psychosom Res $58: 335-342$

6. Tjemsland L, Soreide JA, Malt UF (1998) Posttraumatic distress symptoms in operable breast cancer III: status one year after surgery. Breast Cancer Res Treat 47:141-151

7. Bardwell WA, Natarajan L, Dimsdale JE et al (2006) Objective cancer-related variables are not associated with depressive symptoms in women treated for early-stage breast cancer. J Clin Oncol 24: 2420-2427

8. Claus EB, Schildkraut JM, Thompson WD et al (1996) The genetic attributable risk of breast and ovarian cancer. Cancer 77:2318-2324

9. Narod SA, Offit K (2005) Prevention and management of hereditary breast cancer. J Clin Oncol 23:1656-1663

10. Meiser B (2005) Psychological impact of genetic testing for cancer susceptibility: an update of the literature. Psychol Oncol 14:1060-1074

11. Lodder L, Frets PG, Trijsburg RW et al (2001) Psychological impact of receiving a BRCA1/BRCA2 test result. Am J Med Genet 98:15-24

12. Van Oostrom I, Meijers-Heijboer H, Duivenvoorden HJ et al (2007) Prognostic factors for hereditary cancer distress six months after BRCA1/2 or HNPCC genetic susceptibility testing. Eur J Cancer 43:71-77

13. Schlich-Bakker KJ, ten Kroode HFJ, Ausems MGEM (2006) A literature review of the psychological impact of genetic testing on breast cancer patients. Patient Educ Couns 62:13-20

14. Van Roosmalen MS, Stalmeier PFM, Verhoef LCG et al (2004) Impact of BRCA1/2 testing and disclosure of a positive test result on women affected and unaffected with breast or ovarian cancer. Am J Med Genet 124A:346-355

15. Bonadona V, Saltel P, Desseigne F et al (2002) Cancer patients who experienced diagnostic genetic testing for cancer susceptibility: reactions and behavior after the disclosure of a positive test result. Cancer Epidemiol Biomarkers Prev 11:97-104
16. Schwartz MD, Lerman C, Brogan B et al (2004) Impact of BRCA1/BRCA2 counseling and testing on newly diagnosed breast cancer patients. J Clin Oncol 22:1823-1829

17. Schwartz MD, Lerman C, Brogan B et al (2005) Utilization of BRCA1/BRCA2 mutation testing in newly diagnosed breast cancer patients. Cancer Epidemiol Biomarkers Prev 14:10031007

18. Weitzel JN, McCaffrey SM, Nedelcu R et al (2003) Effect of genetic cancer risk assessment on surgical decisions at breast cancer diagnosis. Arch Surg 138:1323-1328

19. Meijers-Heijboer H, Brekelmans CTM, Menke-Pluymers M et al (2003) Use of genetic testing and prophylactic mastectomy and oophorectomy in women with breast or ovarian cancer from families with a BRCA1 or BRCA2 mutation. J Clin Oncol 21:1675-1681

20. Van Oostrom I, Meijers-Heijboer H, Lodder LN et al (2003) Long-term psychological impact of carrying a BRCA1/2 mutation and prophylactic surgery: a 5-year follow-up study. J Clin Oncol 21:3867-3874

21. Schlich-Bakker KJ, Wárlám-Rodenhuis CC, Van Echtelt $\mathrm{J}$ et al (2006) Short-term psychological distress in patients actively approached for genetic counseling after diagnosis of breast cancer. Eur J Cancer 42:2722-2728

22. Ardern-Jones A, Kenen R, Eeles R (2005) Too much too soon? Patients and health professionals' views concerning the impact of genetic testing at the time of breast cancer diagnosis in women under the age of 40. Eur J Cancer Care 14: 272-281

23. Van Dijk S, Timmermans DR, Meijers-Heijboer H et al (2006) Clinical characteristics affect the impact of an uninformative DNA test result: the course of worry and distress experienced by women who apply for genetic testing for breast cancer. J Clin Oncol 24:3672-3677

24. Schreurs PJ, Van de Willige G, Tellegen B et al (1988) Utrechtse Copinglijst. Omgaan met problemen en gebeurtenissen. Swets \& Zeitlinger, Lisse

25. Westbrook MT (1979) A classification of coping behavior based on multi-dimensional scaling of similarity ratings. J Clin Psychol 35:407-410

26. Bleiker EM, van der Ploeg HM, Hendriks JH et al (1996) Personality factors and breast cancer development: a prospective longitudinal study. J Natl Cancer Inst 88:1478-1482

27. Sprangers MA, Groenvold M, Arraras JI et al (1996) The European Organization for Research Treatment of Cancer breast cancer specific quality-of-life questionnaire module: first results from a three country field study. J Clin Oncol 14:2756-2768

28. Zigmond AS, Snaith RP (1983) The Hospital Anxiety and Depression Scale. Acta Psychiatr Scand 67:361-370

29. Razavi D, Delveaux N, Farvacques C et al (1990) Screening for adjustment disorders and major depressive disorders in cancer in-patients. Br J Psychiatry 156:79-83

30. Horowitz M, Wilner N, Avarez W (1979) Impact of event scale: a measure of subjective stress. Psychosom Med 41:209-218

31. Horowitz MJ (1982) Stress response syndromes and their treatment. In: Goldenberger L, Breznitz S (eds) Handbook of stress. Theoretical and clinical aspects. Free Press, New York

32. Neal LA, Busuttil W, Rollins J et al (1994) Convergent validity of measures of post-traumatic stress disorder in a mixed military and civilian population. J Trauma Stress 7:447-455

33. Coyne J, Kruus L, Racioppo M et al (2003) What do ratings of cancer - specific distress mean among women at high risk of breast and ovarian cancer? Am J Med Genet 116:222-228

34. Gurevich M, Devins GM, Wilson C, McCready D et al (2004) Stress response syndromes in women undergoing mammography: a comparison of women with and without a history of breast cancer. Psychosom Med 66:104-112 